\title{
The Fuzzy-Sets Theoretic Interpretation of Fibonacci Numbers and Golden Section
}

\author{
Faramarz Faghihi \\ Bioinformatics Laboratory, Nanobiotechnology Research Center \\ Avesina Research Institute, ACECR, Tehran, Iran \\ faghihi@avesina.ac.ir
}

\begin{abstract}
The Fibonacci numbers and golden section were found to be present in numerous applications and processes, from the design of our bodies to the structure of DNA. We introduce Fibonacci numbers as fuzzy sets. Such transforming allows further applications of fuzzy sets theory in Fibonacci numbers and related topics. In this paper, three propositions are presented by using the concept of fuzzy entropy and similarity of fuzzy sets.
\end{abstract}

Mathematical classification: 03E72, 11B39

Keywords: Fibonacci numbers, golden section, fuzzy set, fuzzy entropy, similarity of fuzzy set

\section{Introduction}

Mathematics can be considered as the underlying order of the universe, and the Fibonacci numbers and golden section are two of the most fascinating discoveries made in the mathematical world since the time of Archimedes [2,3]. 
The connection between golden section and Fibonacci numbers is well known.

In this section we include some elementary aspects that are necessary for this paper.

Definition 1.1. Fibonacci numbers starts with 0 and 1, and then produces the next Fibonacci number by adding the two previous Fibonacci numbers.

Fibonacci number are $0,1,1,2,3,5,8,13, \ldots$

and are defined by

$$
f_{0}=1, f_{1}=1, f_{n}=f_{n-1}+f_{n-2} \quad \text { for } \quad n \geq 2 \quad n \in N
$$

Definition 1.2. In mathematics, two quantities are in the golden section if the ratio between the sum of those quantities and the larger one is the same as the ratio between the larger one and the smaller. The golden ratio is approximately 1.6180339887.

\section{C}

A

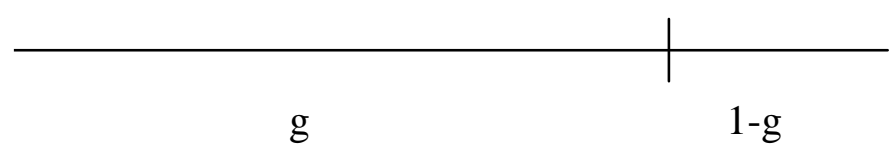

B

$\frac{1-g}{g}=\frac{g}{1} \quad$ or $\quad g^{2}+g-1=0 \Rightarrow \mathbf{g}=\frac{(\sqrt{5})-1}{2}=0.6180339$

The length of $A B$ is unit.

$g$ is called the golden section and is shown by $\Phi$.

$\Phi=\frac{1}{\varphi}=0.6180339$

$\varphi=1.6180339$

The equation of golden section is $\phi+\phi-1=0$ 
The connection between golden section and Fibonacci numbers is well known.

Johannes Kepler pointed out that the ratio of consecutive Fibonacci numbers converges to the golden ratio as the limit

$\lim \frac{F_{n+1}}{F_{n}}=\varphi \quad \lim \frac{F_{n}}{F_{n \rightarrow \infty}}=\Phi$

\section{Fibonacci numbers as ordered fuzzy sets}

Fuzzy sets theory is the extension of classical sets theory [5]. A fuzzy set is defined as follows.

Definition 2.1. Let $X$ be a set, $X=\left\{x_{1}, x_{2}, \ldots, x_{n}\right\}$. A fuzzy set in $X$ is given by

$$
A=\left\{\left(x, \mu_{A}(x)\right) \mid x \in X\right\}, \quad \mu_{A}(x) \in[0,1] .
$$

The membership function $\mu_{A}(x) \in[0,1]$ is such that $\mu_{A}(x)$ quantifies the grade of membership of the element ${ }^{x}$ of $X$.

Definition 2.2. The geometric definition of fuzzy entropy of any fuzzy set $P[1]$ is

$$
\operatorname{Ent}(P)=\frac{\left|P-P_{\text {near }}\right|}{\left|P-P_{\text {far }}\right|} \quad[0,1]
$$

and Clarity $=1-$ Entropy

where $P_{\text {near }}$ and $P_{\text {far }}$ are closest and furthest crisp set to $P$ respectively.

Definition 2.3. A set-theoretic operation based similarity measure is defined [4] as Simil $(P, Q)=\frac{P \cap Q}{P \bigcup Q}$ 
Here $\mathrm{P}$ and $\mathrm{Q}$ are two fuzzy sets

$P=\left(p_{1}, p_{2}, \ldots, p_{n}\right) \quad Q=\left(q_{1}, q_{2}, \ldots, q_{n}\right)$

where $\cap$ and $U$ are intersection and union of fuzzy sets respectively. Using the min and max operators for intersection and union of fuzzy sets respectively

$\operatorname{Simil}(P, Q)=\frac{\sum_{i=1}^{n} \min \left\{p_{i}, q_{i}\right\}}{\sum_{i=1}^{n} \max \left\{p_{i}, q_{i}\right\}}$

To define Fibonacci numbers as fuzzy set we consider the Fibonacci numbers as the ratio of

$\frac{F_{n}}{F_{n+1}}: 1,1 / 2,2 / 3,3 / 5,5 / 8,8 / 13, \ldots \quad n=1,2, \ldots$

and also

$\left(1-\frac{F_{n}}{F_{n+1}}\right): 0,1 / 2,1 / 3,2 / 5,3 / 8,5 / 13, \ldots n=1,2, \ldots$

For each $n$, we define a fuzzy set as a string composed of two elements

$$
\begin{aligned}
P_{n} & =\left(\frac{F_{n}}{F_{n+1}}, 1-\frac{F_{n}}{F_{n+1}}\right) \\
& =\left(\frac{F_{n}}{F_{n+1}}, \frac{F_{n+1}-F_{n}}{F_{n+1}}\right)
\end{aligned}
$$

Fibonacci numbers $=\left(p_{1}, p_{2}, \ldots, p_{n}\right)$

So

we

have

Transformed Fibonacci numbers $=(1,0),(1 / 2,1 / 2),(2 / 3,1 / 3),(3 / 5,2 / 5),(5 / 8,3 / 8),(8 / 13,5 / 13), \ldots$

Proposition 2.1. Fuzzy entropy of $P_{n+1}$ is equal to the golden section when $n$ goes to infinity.

Proof. For defined fuzzy sets nearest and furthest crisp sets are 
$P^{\text {near }}=(1,0)$ and $\quad P^{\text {far }}=(0,1)$

Using the relation (4)

$\operatorname{Ent}\left(P_{n+1}\right)=\frac{1-\frac{F_{n+1}}{F_{n+2}}+\frac{F_{n}}{F_{n+2}}}{\frac{F_{n+1}}{F_{n+2}}+1-\frac{F_{n}}{F_{n+2}}}=\frac{\frac{F_{n+2}-F_{n+1}+F_{n}}{F_{n+2}}}{\frac{F_{n+1}+F_{n+2}-F_{n}}{F_{n+2}}}$

Using the relation $F_{n+2}-F_{n+1}=F_{n}$

we have

$\operatorname{Ent}\left(P_{n+1}\right)=\frac{F_{n}}{F_{n+1}}$

Using the relation ( 3 )

$\lim \operatorname{Ent}\left(P_{n+1}\right)=\lim \frac{F_{n}}{F_{n+1}}=\Phi$

$n \rightarrow \infty \quad n \rightarrow \infty$

Proposition 2.2. For each fuzzy set $P_{n}, P_{n}=\left(\operatorname{Ent}\left(P_{n+1}\right), \operatorname{Clar}\left(P_{n+1}\right)\right)$

Proof. From Preposition 1, we know that

$\operatorname{Ent}\left(P_{n+1}\right)=\frac{F_{n}}{F_{n+1}} \quad$ and $\quad \operatorname{Clar}\left(P_{n+1}\right)=\frac{F_{n+1}-F_{n}}{F_{n+1}}$

Regarding the relation ( 7 )

$P_{n}=\left(\operatorname{Ent}\left(P_{n+1}\right), \operatorname{Clar}\left(P_{n+1}\right)\right)$

Proposition 2.3. Similarity of two fuzzy sets $P_{n}$ and $P_{n+1}$ when $n$ goes to infinity, is equal to 1.

Proof. If we consider two consequently fuzzy sets of the string $P_{n}$ and $P_{n+1}$

$$
P_{n}=\left(\frac{F_{n}}{F_{n+1}}, \frac{F_{n+1}-F_{n}}{F_{n+1}}\right) \quad P_{n+1}=\left(\frac{F_{n+1}}{F_{n+2}}, \frac{F_{n+2}-F_{n+1}}{F_{n+2}}\right)
$$

Using the similarity definition (6) 
$\operatorname{Similarity~}\left(P_{n}, P_{n+1}\right)=\frac{\sum_{i=1}^{2} \min \left(p_{n}^{i}, p_{n+1}^{i}\right)}{\sum_{i=1}^{2} \max \left(p_{n}^{i}, p_{n+1}^{i}\right)}=\frac{\frac{F_{n}}{F_{n+1}}+\frac{F_{n}}{F_{n+2}}}{\frac{F_{n+1}}{F_{n+2}}+\frac{F_{n+1}-F_{n}}{F_{n+1}}}$

$$
=\frac{F_{n} \cdot\left(2 \cdot F_{n+1}+F_{n}\right)}{F_{n+1}^{2}+\left(F_{n+1}^{2}-F_{n}^{2}\right)}
$$

Similarity $\left(P_{n}, P_{n+1}\right)=\frac{F_{n}^{2}+2 \cdot F_{n} \cdot F_{n+1}}{F_{n+1}{ }^{2}-F_{n}{ }^{2}}$

By dividing on $F_{n}{ }^{2}$, we get

similarity $\left(P_{n}, P_{n+1}\right)=\frac{1+2 \frac{F_{n+1}}{F_{n}}}{2\left(\frac{F_{n+1}}{F_{n}}\right)^{2}-1}$

We know that

$$
\lim _{n \rightarrow \infty} \frac{F_{n+1}}{F_{n}}=\frac{1}{\Phi}
$$

Therefore

$$
\lim \frac{1+2 \cdot \frac{F_{n+1}}{F_{n}}}{2 \cdot\left(\frac{F_{n+1}}{F_{n}}\right)^{2}-1}=\frac{\Phi^{2}+2 \Phi}{2-\Phi^{2}}
$$

Regarding that

$$
\Phi^{2}-\Phi-1=0
$$


$\Phi^{2}+2 \Phi=2-\Phi^{2}$

We have

$\lim \operatorname{similarity}\left(P_{n}, P_{n+1}\right)=1$

$n \rightarrow \infty$

It means that the similarity of two consequent fuzzy sets when $n$ goes to infinity is equal to one.

\section{Conclusions}

In this paper, we showed that considering Fibonacci numbers as a fuzzy set makes it possible to think on Fibonacci numbers and golden section in a new way. Propositions that presented are some preliminary consequences of this transformation. Further works should be done to find more theoretical results and also the applications of these results.

\section{References}

[1] B. Kosko, Neural networks and fuzzy system, Prentice-Hall, Englewood Cliffs, NJ, 1992.

[2] A. Stakhov, The Generalized Principle of the Golden Section and its applications in mathematics, science, and engineering, Chaos, Solitons \& Fractals, 26 ( 2005), 263-289.

[3] A. Stakhov, Fundamentals of a new kind of Mathematics based on the Golden Section, Chaos, Solitons \& Fractals, 27 ( 2005), 1124-1146. 
[4] M. Setnes, R. Babuska, U. Kaymak, H.R.N. Lemke, Similarity Measures in Fuzzy Rule Base Simplification, IEEE Trans on Systems Man and Cybernetics. 28 (1998), 376-386.

[5] L.A. Zadeh, Fuzzy Sets, Information and Control, 8 (1965) 338-352.

Received: August 6, 2007 